\title{
Retraction of: Effects of Photobiomodulation Therapy on Patients with Primary Open Angle Glaucoma: A Pilot Study (DOI: 10.1089/pho.2015.3944)
}

The online-ahead-of-print published article, "Effects of Photobiomodulation Therapy on Patients with Primary Open Angle Glaucoma: A Pilot Study," by Ivandic BT and Ivandic T (DOI: 10.1089/pho.2015.3944), is being officially retracted from Photomedicine and Laser Surgery due to potentially significant errors in the reported parameters and calculated doses.

The second author of the article, Dr. Tomislav Ivandic, notified the Journal that inaccuracies in the reported parameters were discovered after publication of the article as a consequence of the information being translated from German (the authors' native language) into English. As a very serious repercussion of this error, Dr. Ivandic states that, "Further studies or application based on these false doses would probably harm the eye."

The first-named author of the article, Dr. Boris Ivandic, disagrees with this declaration and states that, "there is no mistake in the dose calculations using the parameters $10 \mathrm{~mW}$ power, $3 \mathrm{~mm}^{2}$ beam spot area as well as 30 or $90 \mathrm{sec}$ time of irradiation, respectively."

Photomedicine and Laser Surgery is dedicated to upholding the integrity of the science it publishes. As a result of this irreconcilable dispute between the authors, the editorial leadership of the Journal is officially retracting this article from the literature in an effort to reduce any potential for iatrogenic injury in the event that the erroneous information was used to study or treat other patients.

The authors regret this very unfortunate circumstance. 